\title{
A Scale Model of Tension Structures in Road Tunnels to Optimize the Use of Solar Light for Energy Saving
}

\author{
Antonio Peña-García, ${ }^{1}$ Luisa-María Gil-Martín, ${ }^{2}$ Roberto Escribano, ${ }^{3}$ \\ and Antonio Espín-Estrella ${ }^{1}$ \\ ${ }^{1}$ Department of Civil Engineering, ETSICCP, University of Granada, 18071 Granada, Spain \\ ${ }^{2}$ Department of Structural Mechanics, ETSICCP, University of Granada, 18071 Granada, Spain \\ ${ }^{3}$ Department of Graphical Expression in Architecture and Engineering, ETSIE, University of Granada, 18071 Granada, Spain
}

Correspondence should be addressed to Antonio Peña-García, pgarcia@ugr.es

Received 25 May 2011; Revised 30 June 2011; Accepted 13 July 2011

Academic Editor: Jimmy Yu

Copyright (C) 2011 Antonio Peña-García et al. This is an open access article distributed under the Creative Commons Attribution License, which permits unrestricted use, distribution, and reproduction in any medium, provided the original work is properly cited.

\begin{abstract}
Shifting the threshold zone of road tunnels with semitransparent tension structures has shown itself to be an effective way of saving energy in regards to electricity consumption, maintenance, and construction materials used in the electrical lighting, thus lessening negative environmental impacts. Even though the shape of the tension structure has a major influence on energy savings, the optimal type of structure for each tunnel is often difficult to determine, because experiments using real tunnels are extremely expensive. It is thus necessary to find methods of doing this that are both reliable as well as economical. In this research study, three candidate structures were set up at the portal of a scale model of a real tunnel. The energy savings in each case were analyzed and compared. As a result, it was possible to formulate a new equation that calculates the energy savings in the threshold zone.
\end{abstract}

\section{Introduction}

Road tunnels are divided into various zones for lighting purposes. Depending on the distance of these zones from the entrance or to the exit of the tunnel, they have different requirements for luminance, $L$ (luminous flux emitted per unit of surface and solid angle in a given direction) and illuminance, $E$ (luminous flux received per unit of surface). A great deal of research has focused on the best way to calculate both luminance and illuminance in road tunnels [1-4], and there are important contributions still being made in this area [5].

These road tunnel zones are the following: (i) access zone (the opencast sky zone just before the beginning of the tunnel), (ii) threshold zone (the first section of the tunnel), (iii) transition zone (the section after the threshold zone), interior zone (the inner section of the tunnel), and (iv) exit zone (the last section before the tunnel ends). A more detailed description of road tunnel zones can be found in [4].

Figure 1 shows that the threshold zone $\left(\mathrm{L}_{\text {th }}\right)$ requires more light than any other section of the tunnel. This means that energy consumption in this section can be extremely high, especially in tunnels where electrical lighting operates 24 hours a day and 365 days a year.

For this reason, a major priority is to reduce energy consumption in the threshold zone, but without negatively affecting the visual capacity of drivers and reducing traffic safety. An effective way of attaining this goal is to shift the threshold zone outside of the tunnel. This affords the possibility of collecting and using sunlight to reach the required illuminance levels instead of doing this solely with electrical lighting. For this purpose, semitransparent tension structures have proven to be an effective solution as shown in other studies $[6,7]$. However, these studies only focused on showing the value of tension structures as an energy-saving mechanism in road tunnels and did not analyze how they could be optimized.

Permanent tension structures have the advantage of being aesthetic, light-weight, flexible, durable, and easy to construct. If they are properly installed, the fabric and auxiliary components will have a useful service life of 30 years 


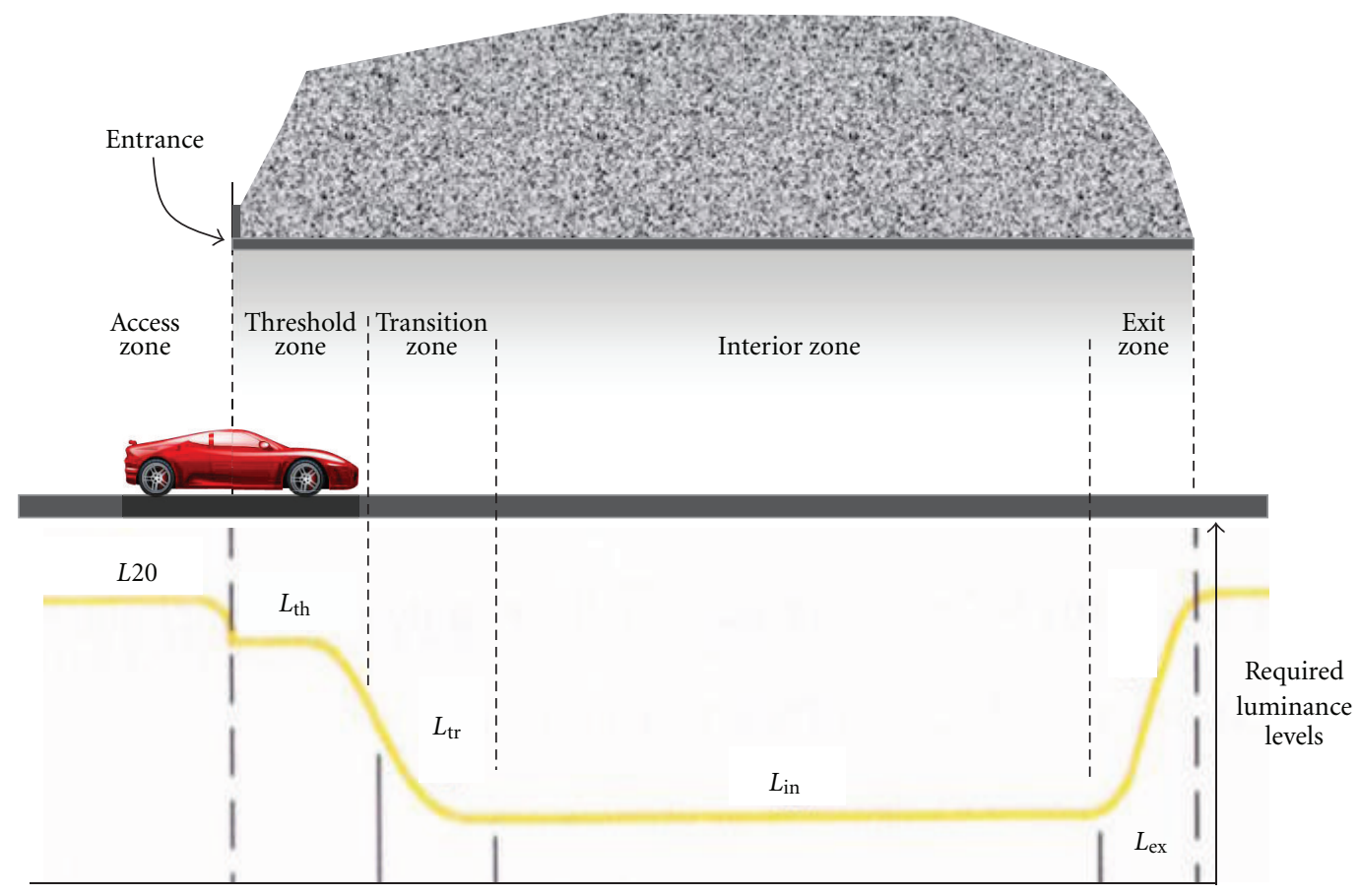

FIgUre 1: Tunnel zones and luminance values.

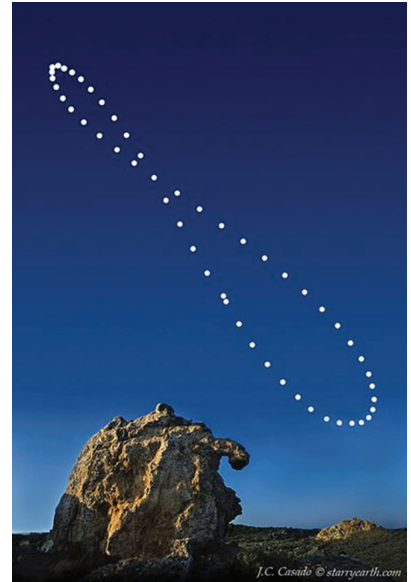

Figure 2: Solar annalema in Cabo de Creus (Gerona, Spain). Juan Carlos Casado: http://tierrayestrellas.com/.

with only routine maintenance. Thanks to their versatility, tensile structures can be adapted to any type of boundary and landscape conditions. If the contour stiffness of their textile material and the positions of their anchoring nodes are defined for a given tunnel, it is then possible to find an equilibrium shape $[8,9]$. In this research study, this shape was defined by a software application developed at the Structural Mechanics Department of the University of Granada.

In order to account for geometrical changes, time-dependent properties (e.g., ageing, creep, and relaxation), and loading conditions (e.g., snow and wind), second-order structural analysis is required. Engineers must consider that the tension structure has to be able to effectively eliminate snow and water. To ensure the durability of the permanent structure, the textile material is covered with a long-lasting PVC coating that increases its resistance to UV radiation. This coating also has dirt-repellent properties that improve the performance of the fabric, which is flame retardant.

Nevertheless, in spite of the mechanical performance of fabric structures and their successful implementation in road tunnels, their energy-saving features must be optimized in order to maximize benefits. Since previous research has only focused on proving the effectiveness of these structures for saving energy in road tunnels, the design pattern used was invariably the simplest, in other words, flat and rectangular. Factors pertaining to shape (e.g., curvature, symmetry, etc.) were not considered. However, this study has gone a step farther, since it evaluates the influence of the structure's shape on its energy-saving properties in a road tunnel. Shape impact was found to be important, particularly in tunnels at a certain location and with a certain orientation. Apart from the shape of the tension structure, other factors that affect the amount of solar power transmitted by it are the following.

(i) Tunnel location: the latitude and longitude of the tunnel determine how and how much luminous flux of solar light can be collected. Depending on the tunnel location, the sun rays will be more or less perpendicular. Since more perpendicular rays carry more energy, the way that tension structures transmit solar light at locations and times when the sun is very high must be maximal. Figure 2 shows the extreme variability of solar position during the year.

(ii) Tunnel orientation: at a certain location with a given pattern of solar radiation during the year, the illuminance on the access zone of a tunnel will depend very 
much on the orientation of the tunnel. Thus, a tunnel entrance with an east-west orientation will receive more illuminance in the morning, because in the afternoon, the sun might be behind the mountain. If the orientation of the tunnel is south-north, then the side receiving more light will depend on the hour of the day.

(iii) Material of the structure: given that solar light must be transmitted by the tension structure and reach the road below, the material of the structure is also crucial for saving energy because of its transmittance. Nevertheless, it cannot be arbitrarily transparent. If the illuminance under the structure is very high, the driver's eye will not begin to adapt properly to the darkness. This means that the shift of the threshold zone to the outside of the tunnel will have been useless. In our research study, we have used polyester structures with PVC coating, which give very good results from both an energy and mechanical (i.e., resistance and maintenance) perspective. In future work, we plan to test other materials, once we have fully optimized the shape of the tension structure in relation to the other tunnel parameters (e.g., orientation and location).

Consequently, the choice of an optimal tension structure for the portal of a tunnel is extremely important, because it can lead to significant energy savings. This choice depends on a wide range of factors such as the tunnel's orientation, its, location in relation to the sun's path, road width, and roughness of the landscape. This study is a starting point for what may become standard construction design considerations in the future.

The objectives of this study are the following:

(i) to measure the illuminance levels under three different scale tension structures installed at the portal of a scale model of a road tunnel and convert the measurements obtained to equivalent values for the actual road tunnel under real conditions of orientation, insolation, time of day, and season of the year,

(ii) to formulate a general equation, based on these results, which can calculate the influence of the tension structures on energy savings for each tunnel, insolation conditions, shape of the structure, and textile material.

This effective tool consists of the combination of a set of measurements obtained from a scale model and the deduced equation. It is a quick and economical method of obtaining the most energy-efficient tension structure to be installed at the portal of road tunnels. The results obtained are presented, analyzed, and discussed in the following sections.

\section{Materials and Methods}

Illuminance under a tension structure is significantly affected by tunnel orientation and location as well as by the shape of the structure. Ideally, it would be necessary to perform experiments in tunnels with all possible orientations and locations in order to determine the most efficient shape of the tension structure in each case. From a more practical viewpoint, however, this is not possible. Even performing a single field study in a real tunnel is extremely costly in terms of time, money, and bureaucracy. Thus, a model is needed where real-life conditions can be simulated.

For our study, we built a mock-up (scale 1:25), which simulated a long road tunnel with a mechanism that enabled it to adopt all possible orientations in a circle of $360^{\circ}$ (see Figure 3). Above the mock-up, we installed a sun ecliptic model with an Ultra Vitalux $300 \mathrm{~W}$ lamp, which had the same spectral composition as sunlight. By changing the sun's position in relation to the tunnel, it was possible to simulate any tunnel location and orientation. In addition, the light source was able to slide along the ecliptic plane and thus simulate any hour of the day.

As previously mentioned, this simulation of a real tunnel allowed us to determine the most energy-efficient semitransparent tension structure. For this purpose, three structures were tested. The shapes and dimensions of these tension structures were strongly conditioned by the space available at the entrance of the tunnel and the safety distances established in the Spanish highway regulations. In regards to energy efficiency, the optimal place for the anchoring points was determined for each tunnel, depending on its location and orientation. Once these anchoring points were defined, a final optimization was performed in order to obtain the simplest pattern design, and thus, the most cost-efficient structure.

The three structures studied were designed so that the equilibrium shapes had sufficient slope to ensure that there would be no accumulation of material and to facilitate the elimination of rainwater drag. This meant that only low maintenance was necessary.

The typical fabrics used in outdoor structures are polyester, coated with PVC (polyvinyl chloride), or glass cloth, coated with PTFE (polytetrafluoroethylene, commonly known as Teflon). All the structures in our study were made of polyester with a vanilla-colored PVC coating because of its relatively lower cost $\left(70 €-100 €\right.$ per $\mathrm{m}^{2}$ less than PTFE coating) and also because of the good transmittance values obtained for it in laboratory tests [6].

Even though road lighting is usually designed in terms of the luminance emitted by the road because of electrical lighting, our research results are presented in terms of the illuminance reaching the road underneath the structure. Since our objective was to quantify the energy savings obtained with the tension structure, illuminance was considered more accurate than luminance because of its nondirectionality and also because road luminance involves the reflective properties of the road itself. These properties can affect the evaluation of the amount of light transmitted by the tension structure.

The illuminance under the tension structure was measured at six-centimetre intervals with an HT172 digital luxmeter. We decided to simulate June 21st, the most unfavourable day of the year because of the perpendicularity of solar rays at the location of the simulated tunnel (Jaén, 


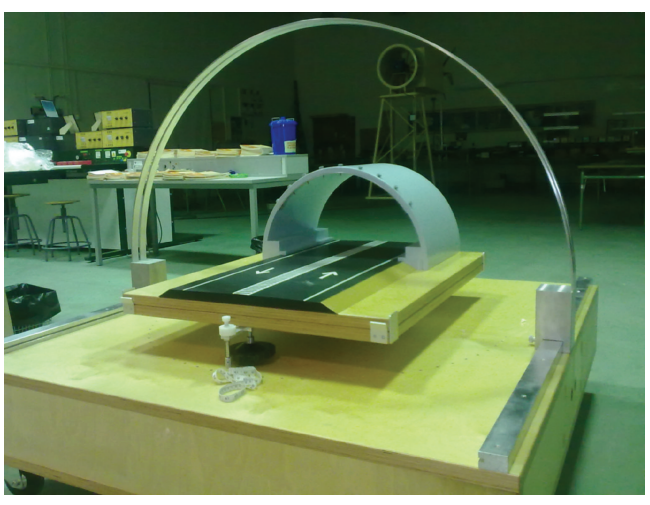

(a)

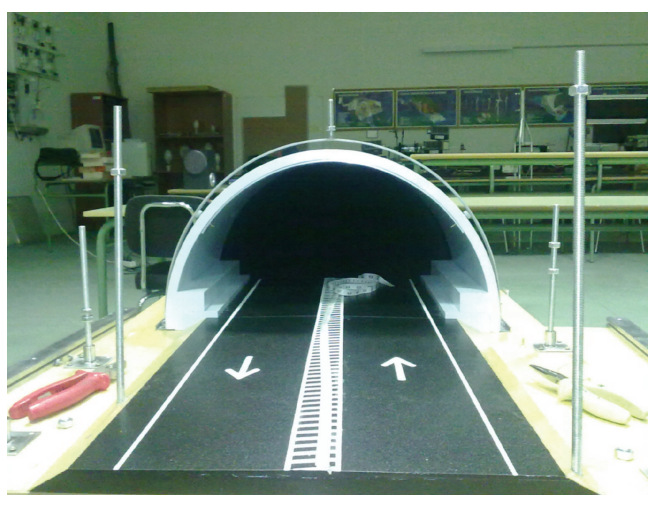

(b)

FIGURE 3: Mock-up of tunnel and the solar ecliptic plane.

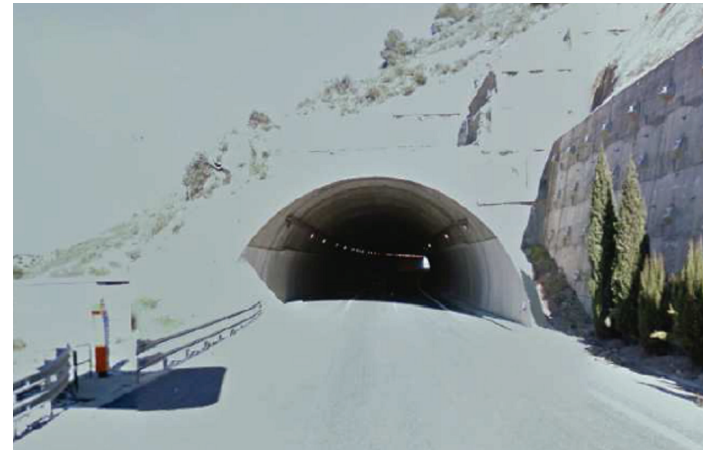

Figure 4: Modelled road tunnel in Pegalajar (Jaén, Spain) with an almost perfect south-north orientation $\left(349.41^{\circ}\right)$.

Spain) (see Figure 4). The insolation conditions were simulated at 12:00, 14:15, and 16:00. The most critical time of the day was $14: 15$, because that is when the sun reaches its highest point at the tunnel location $\left(37.11^{\circ} \mathrm{N}, 3.35^{\circ} \mathrm{W}\right.$, and $683 \mathrm{~m}$ above sea level).

For the purposes of our study, we considered direct sunlight as well as diffuse light or light from the rest of the sky. Diffuse light was taken into account by covering the tension structure with an opaque board and measuring the illuminance underneath the structure. Both components were added though for the sake of simplicity, only the final results are shown.

\section{Results}

Three tension structures were placed at the entrance of the simulated tunnel. The first structure was located on the perimeter of the cross-section of the tunnel, whereas the other two, which were more asymmetrical, were placed at three anchoring points on the outside part of the tunnel behind the entrance. These two asymmetrical structures had a similar shape with the difference that the third structure, anchored at the highest point was placed further behind the entrance of the tunnel than the second one, and the position of the anchoring points was slightly higher.
Since pattern cutting is not a simple process, the choice of design was influenced by the simplicity of shape. The goal was to obtain a simple pattern that would facilitate construction and maintenance. Structures 1, 2, and 3 are shown in Figure 5.

Figure 6 represents the illuminance values in the threshold zone of: (i) a tunnel with no tension structure and (ii) a tunnel with a tension structure. As can be observed, in the first case, the beginning of the threshold zone is located at the tunnel entrance (see Figure 1). However, in the second case, the beginning of the threshold zone is located at the beginning of the tension structure. The shaded area in Figure 6 represents the contribution of natural light to the lighting in the threshold zone of the tunnel. This Figure shows that installing a tension structure over the road at the portal of the tunnel helps the driver's eyes to adapt better to the darkness. The horizontal dotted line indicates the value of the required illuminance level in the first half of the threshold zone. This value was obtained by using the L20 method [4] for the maximum speed allowed in the model tunnel, $80 \mathrm{~km} / \mathrm{h}$. ( $\mathrm{L}_{\text {th }}$ in Figure 1$)$.

The illuminance values under the tension structure (threshold zone) were measured in the laboratory using a fully cosine-corrected HT 172 digital luxmeter. The sun was modelled with an Ultra Vitalux $300 \mathrm{~W}$ lamp, whose radiation spectrum is similar to the sun's. The illuminance values obtained with the model had to be corrected so that they could be extrapolated to the actual values that would have been obtained in the real tunnel illuminated by the sun. This correction was performed by multiplying these values by the correction factors arising from the radiation flux on the Earth's surface. These correction factors are shown in Table 1.

Figure 7 lists the illuminance values measured at the centre of both the right and left road lanes. These values are represented, depending on the distance of the tension structure to the entrance portal for the three times shown in Table 1. (The textile structure was $15 \mathrm{~m}$ long.) In Figure 7, the illuminance values correspond to actual values (i.e., tested values in the mock-up and corrected by the coefficients in Table 1). The origin of the abscissa axis in Figure 7 corresponds to the point at which the tension structure is 


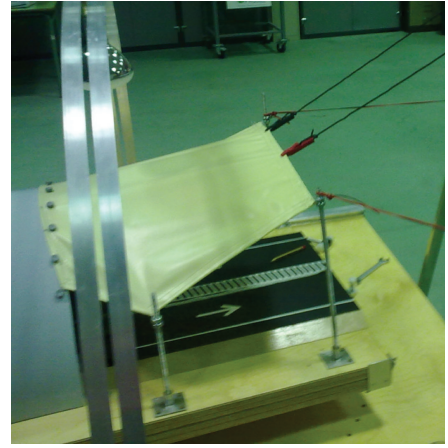

(a)

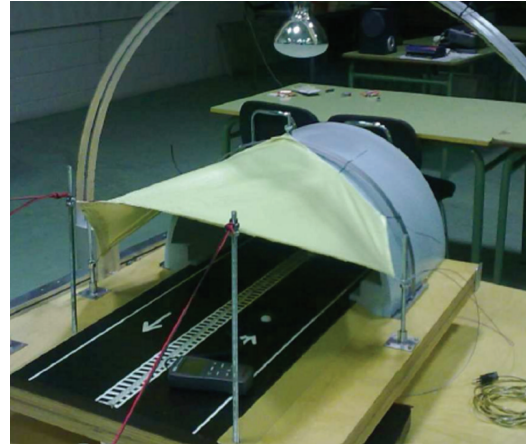

(b)

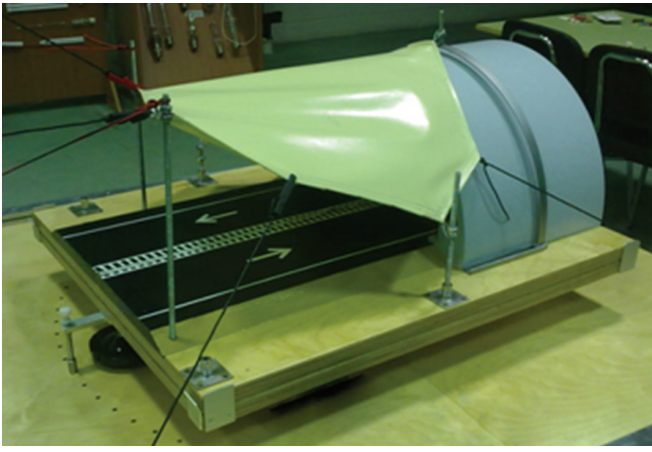

(c)

FIgURE 5: Tension structures placed at the entrance of the tunnel. (a) Structure type 1. (b) Structure type 2. (c) Structure type 3.

TABLE 1: Correction factors used to convert mock-up values into Earth surface values.

\begin{tabular}{|c|c|c|c|}
\hline $\begin{array}{l}\text { Times of the day (June 21st) } \\
\text { when measurements were } \\
\text { taken) }\end{array}$ & $\begin{array}{l}\text { Irradiance on the } \\
\text { mock-up }\left(\mathrm{W} / \mathrm{m}^{2}\right)\end{array}$ & $\begin{array}{l}\text { Irradiance on the Earth's } \\
\text { surface }\left(\mathrm{W} / \mathrm{m}^{2}\right)\end{array}$ & Correction factor \\
\hline $12: 00$ & 300 & 955.20 & 3.184 \\
\hline $14: 15$ & 300 & 1045.20 & 3.484 \\
\hline $16: 00$ & 300 & 992.24 & 3.307 \\
\hline
\end{tabular}

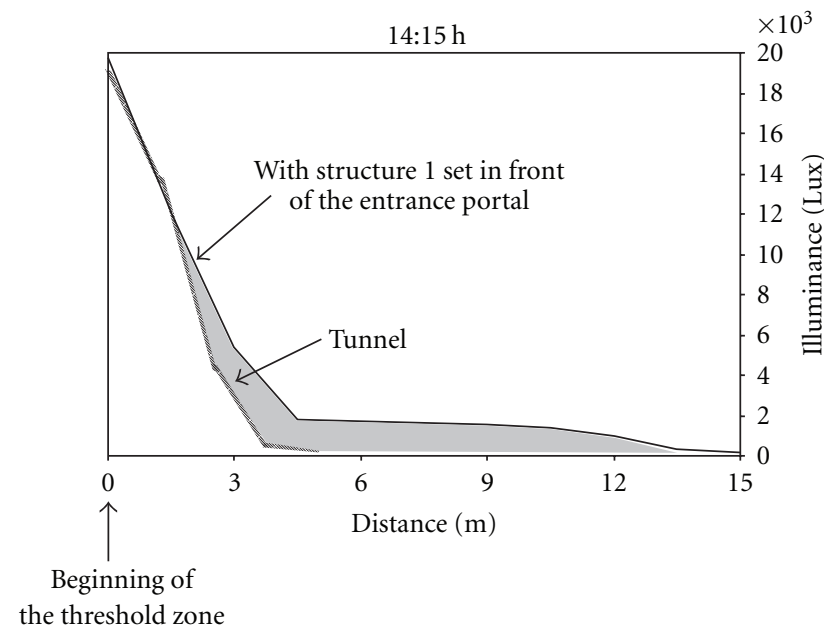

Figure 6: Additional natural light in the threshold zone.

anchored and from which it extends to the centre of the lane. The horizontal dotted lines in Figure 7 correspond to the required illuminance that was calculated for this tunnel with the L20 method for a maximum speed of $80 \mathrm{~km} / \mathrm{h}$ [4].

In Figure 7, the dotted segments in the graphs corresponding to the left lane indicate that the structure does not overlap with the centre of the lane at this point. This is due to the asymmetry of the structure that covers a larger area of the right lane than of the left. Even though the illuminance values corresponding to these dotted lines are represented in Figure 7, they were not considered in the calculations, because they do not lie under the structure. The mean illuminances are shown in Table 2.
Based on the previously described results, potential energy savings under the structure were evaluated for both lanes and for the three peak times and structures. These values are given in Table 3 and are represented in Figure 8. As shown in Figure 8, energy saving is defined as the average of the illuminance values under the tension structure divided by the required illuminance value in the CIE standards. The theoretical mean illuminance value for this tunnel is $E_{m}=$ 8222 lux.

The values for both lanes at the three peak times were averaged. The results are given in Table 4, namely, the energy savings of the three tension structures. Both Table 4 and Figure 8 show that structure 2 was the most energy-efficient and had the most regular performance at the three peak hours.

Although the energy savings achieved with structure 1 were greater for the left lane, our decision regarding the most suitable structure was based on the results obtained for the right lane, because in countries with right-hand traffic, the vast majority of vehicles circulate in this lane at tunnel entrances. Obviously, in countries with left-hand traffic, such as England, Japan, and Australia, the asymmetry of the structures would be the inverse.

3.1. A General Expression for the Evaluation of Energy Savings in Tension Structures: The ESTS Equation: Comparison of the Three Tension Structures. The results in Table 4 are even more striking when we calculate the amount of energy saved with each structure as compared to the actual energy consumption in the threshold zone. The total flux in the threshold zone without a tension structure can be 


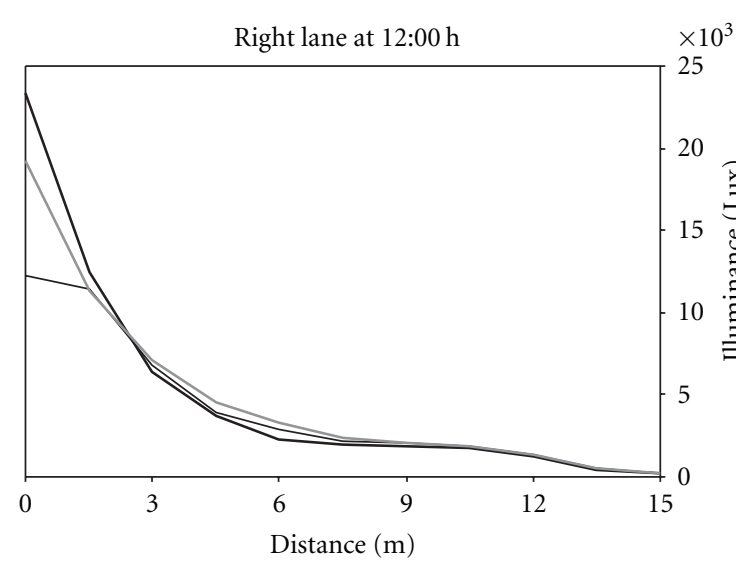

(a)

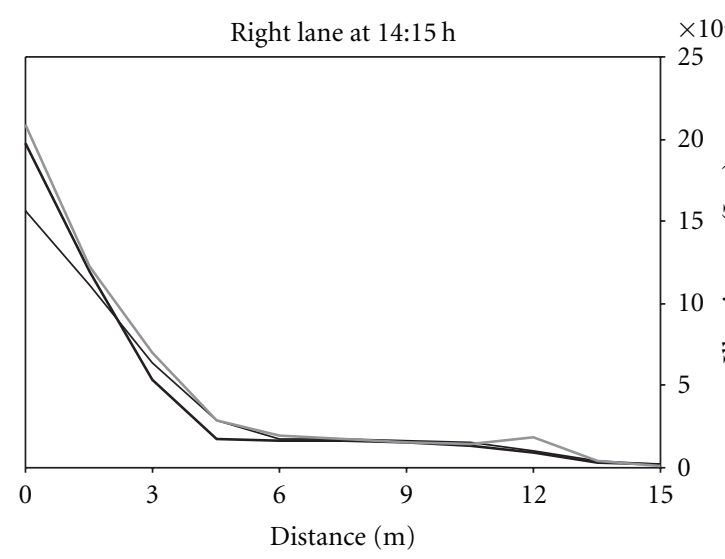

(c)

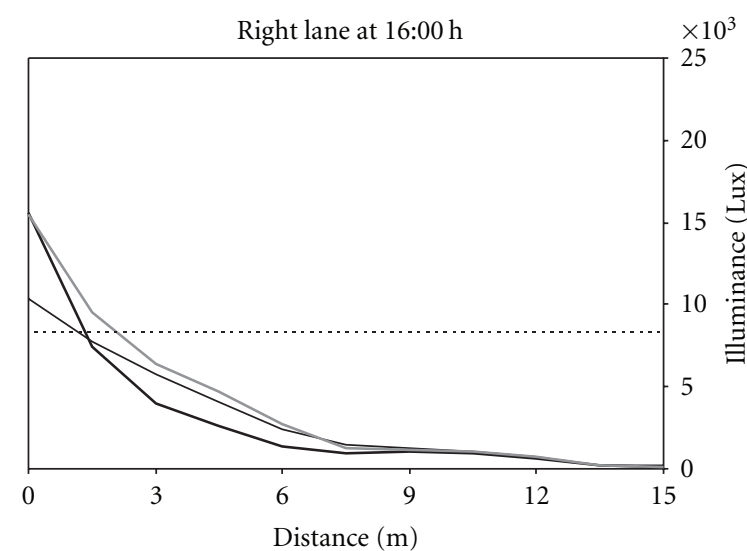

Structure 1
- Structure 2
- Structure 3

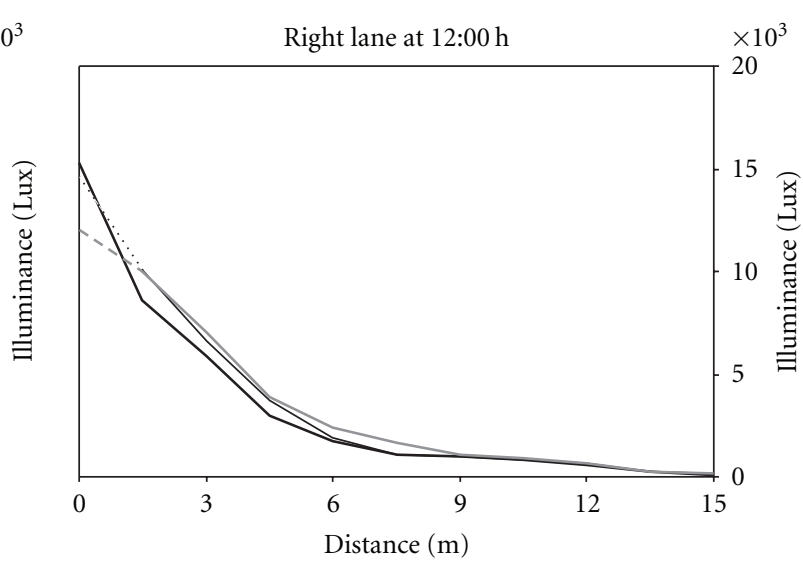

(b)

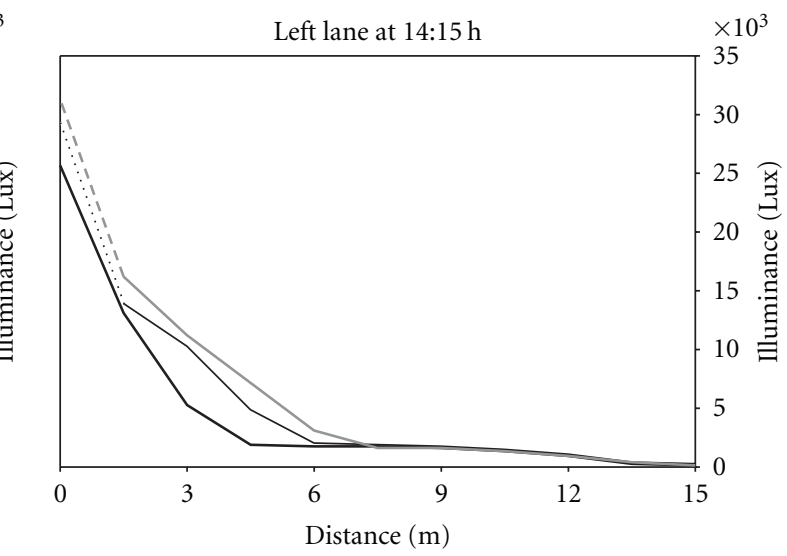

(d)

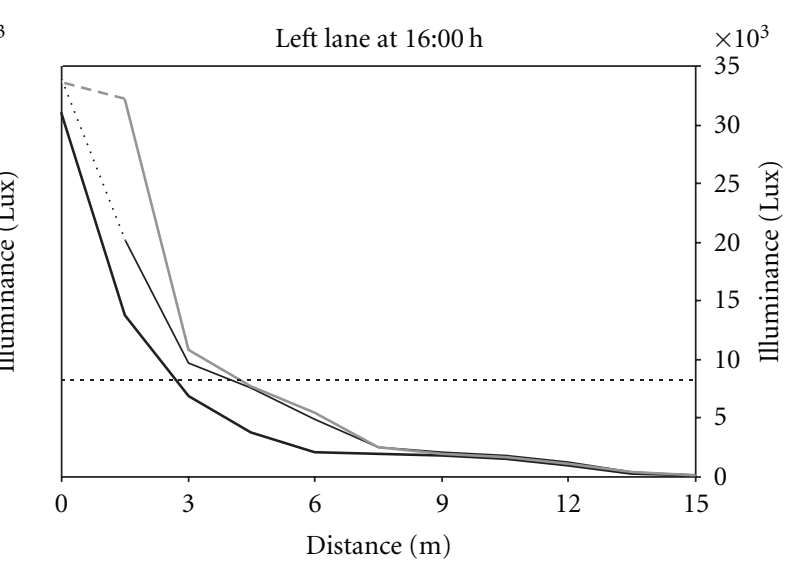

Structure 1
- Structure 2
- Structure 3

(e)

(f)

FIgURE 7: Illuminance values (lux) depending on the distance to the entrance in the centre of the right lane and the centre of the left lane for the N-S tunnel.

obtained with the following expression [10]:

$$
\Phi_{t}=\frac{E_{m} d l_{t}}{2}
$$

where $\Phi_{t}$ is the total luminous flux, $E_{m}$ is the mean illuminance, $d$ is the width of the tunnel, and $l_{t}$ is the length of the threshold zone. In our case, when the threshold zone is shifted by means of a tension structure, (1) becomes the following:

$$
\Phi_{t-S}=\frac{(1-S) E_{m} d l_{S}}{2}+\frac{E_{m} d\left(l_{t}-l_{S}\right)}{2}
$$




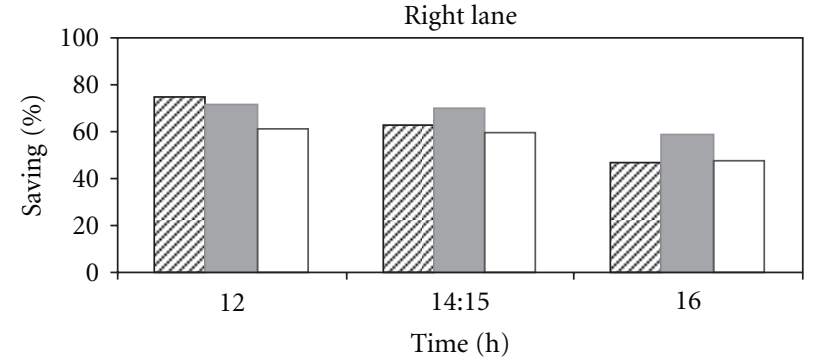

Structure 1

Structure 2

$\square$ Structure 3

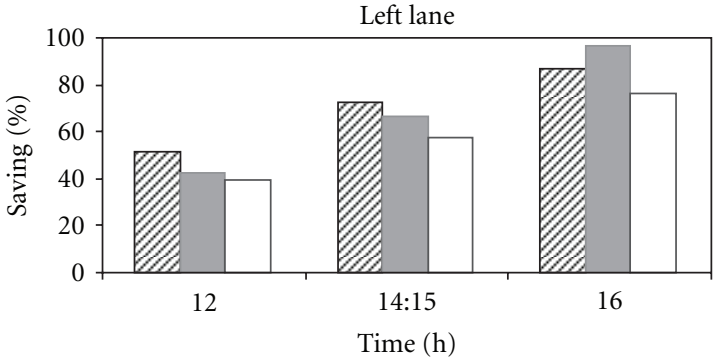

■ Structure 1

- Structure 2

$\square$ Structure 3

(a)

(b)

FIGURE 8: Energy savings in both lanes of the model tunnel for the three tension structures and the three peak hours.

TABLE 2: Illuminance values under the three tension structures at different times of the day.

\begin{tabular}{|c|c|c|c|c|c|c|}
\hline \multirow[b]{2}{*}{ TIME } & \multicolumn{3}{|c|}{ Mean illuminance in lux (right lane) } & \multicolumn{3}{|c|}{ Mean illuminance in lux (left lane) } \\
\hline & $12: 00 \mathrm{~h}$ & $14: 15 \mathrm{~h}$ & $16: 00 \mathrm{~h}$ & $12: 00 \mathrm{~h}$ & $14: 15 \mathrm{~h}$ & $16: 00 \mathrm{~h}$ \\
\hline Structure 1 & 6104 & 5123 & 3845 & 4226 & 5929 & 7120 \\
\hline Structure 2 & 5884 & 5730 & 4784 & 3450 & 5410 & 7939 \\
\hline Structure 3 & 4984 & 4865 & 3878 & 3235 & 4668 & 6266 \\
\hline
\end{tabular}

where $\Phi_{t-S}$ is the luminous flux in the whole threshold zone, including the part under the tension structure, $l_{S}$ is the length of the tension structure, and $S$ is the energy savings determined with the model for the right lane in the case of each structure (see Table 4 ).

When the ratio of the fluxes given by (1) and (2) is obtained, the three tension structures can be compared in relation to a given threshold zone

$$
\frac{\Phi_{t-S}}{\Phi_{t}}=1-S \frac{l_{S}}{l_{t}}
$$

The power consumption of any luminaire is defined as

$$
P=\frac{\Phi}{\eta}
$$

where $\Phi$ is the luminous flux emitted by the luminaire and $\eta$ is the output. Accordingly, and given the fact that the power is the energy consumed per unit of time, (3) can be written as

$$
\frac{Q_{t-S}}{Q_{t}}=1-S \frac{l_{S}}{l_{t}},
$$

where $Q_{t-S}$ is the energy consumed in the threshold zone shifted by a tension structure and $Q_{t}$ is the energy consumed in the same threshold zone without a tension structure.

Equation (5), which is derived from the results of our study, is a new contribution that until now has not been formulated and reported in any research. It yields the electrical energy consumed in a tunnel threshold zone shifted by a tension structure as compared to the electrical energy consumed in a tunnel threshold zone with no tension structure.
Since (5) calculates energy saving under tension structures, we have called it the ESTS equation.

Because the energy consumption is normalized for the total consumption of the threshold zone, (5) can be regarded as a general expression, and thus can be used in the comparison of any tension structure at a tunnel entrance, regardless of its shape or material.

Equation (5) also includes extreme cases. If the whole threshold zone is shifted by the tension structure, $l_{S}=l_{t}$, then $Q_{t-S} / Q_{t}=1-S$. In other words, the consumption in electric lighting is only due to the difference between the actual and theoretical illuminance on the road under the tension structure. On the contrary, if the threshold zone is not shifted using a tension structure, $l_{S}=0$, from (5): $Q_{t-S} / Q_{t}=1$. This means that there is no reduction in electrical lighting consumption.

In the road tunnel modelled in the mock-up, for which the maximum speed permitted is $80 \mathrm{~km} / \mathrm{h}$, the length of the threshold zone, $l_{t}$, is $64 \mathrm{~m}$, and the required illuminance is 8222 lux. The length of the tension structure at the tunnel entrance, $l_{S}$, was $15 \mathrm{~m}$.

For typical high-pressure sodium luminaires of $48000 \mathrm{~lm}$ and $400 \mathrm{~W}$, the annual amount of energy consumed in the threshold zone is 98.11 MWh. Equation (5) can be used to obtain the energy savings per year for each tension structure. Table 5 summarizes these energy savings (MWh) for a mean period of 8 hours of sun per day (the usual period in Spain). Obviously, at night, electric lighting would be necessary to illuminate the road. However, because tunnel lighting at night is much weaker than during the day, low-power luminaries would be sufficient. 
TABLE 3: Energy saving (\%) for three tension structure shapes at the times tested.

\begin{tabular}{lcccccc}
\hline & \multicolumn{3}{c}{ Energy savings for the right lane (\%) } & \multicolumn{3}{c}{ Energy savings for the left lane (\%) } \\
TIME & $12: 00 \mathrm{~h}$ & $14: 15 \mathrm{~h}$ & $16: 00 \mathrm{~h}$ & $12: 00 \mathrm{~h}$ & $14: 15 \mathrm{~h}$ & $16: 00 \mathrm{~h}$ \\
\hline Structure 1 & 74.25 & 62.31 & 46.76 & 51.40 & 72.11 & 86.60 \\
Structure 2 & 71.57 & 69.69 & 58.19 & 41.96 & 65.80 & 96.56 \\
Structure 3 & 60.62 & 59.17 & 47.17 & 39.34 & 56.78 & 76.21 \\
\hline
\end{tabular}

TABLE 4: Average energy savings, $S,(\%)$ under each of the three tension structures.

\begin{tabular}{lcr}
\hline & Energy savings for the right lane (\%) & Energy savings for the left lane (\%) \\
\hline Structure 1 & 61.11 & 70.04 \\
Structure 2 & 66.48 & 68.11 \\
Structure 3 & 55.65 & 57.45 \\
\hline
\end{tabular}

\section{Conclusions}

The shape of a tension structure has a remarkable influence on energy savings when it is placed in front of a tunnel entrance. The research study described in this paper provides a powerful tool that avoids the need to perform experiments in real tunnels with different orientations and locations to determine the most cost-efficient shape of a tension structure. Thanks to this tool, consisting of the combination of a scale model (including the sun, a solar ecliptic, tunnel, and candidate tension structures) and the newly derived ESTS equation, it is possible to determine the optimal design pattern of a tension structure. Needless to say, this saves huge amounts of time, money, and bureaucracy. The results obtained in this study highlight the fact that the effectiveness of a tension structure depends on its having a versatile shape design that takes both aesthetic factors and potential maintenance into account.

In this study, energy savings were found to be different for each road lane because of the asymmetry of the tension structure as well as the orientation, latitude, and longitude of the tunnel. The right lane was used as the reference, since traffic is right-handed in Spain, and vehicles thus entered the tunnel in the right lane.

The results showed that the most suitable semitransparent tension structure for the model tunnel in our study was structure 2 . This procedure can be easily performed in any tunnel in the world given that the model tunnel can take any orientation and the sun ecliptic model can reproduce any latitude and longitude. Consequently, the use of a tension structure to shift the threshold zone to the outside of a road tunnel has shown itself to be an effective way of saving energy. This research provides a model that optimizes the shape of the structure at the portal of the tunnel by comparing it to other tension structures by means of the ESTS equation, which was formulated as part of this re-search. The procedure described is extremely useful, since it quantifies the energy savings of tension structures in MWh, and thus helps engineers to decide which type of structure is most cost effective.
TABLE 5: Energy savings (MWh) in the threshold zone for the three tension structures.

\begin{tabular}{lc}
\hline & Energy savings (MWh) \\
\hline Structure 1 & 4.7 \\
Structure 2 & 5.1 \\
Structure 3 & 4.3 \\
\hline
\end{tabular}

\section{Acknowledgment}

This research was funded by the Spanish Ministry of Education and Science within the framework of Research Project no. BIA 2007-62595.

\section{References}

[1] D. A. Schreuder, "Tunnel entrance lighting: a comparison of recommended practice," Lighting Research and Technology, vol. 3, pp. 274-278, 1971.

[2] W. Adrian, "Investigations on the required luminance in tunnel entrances," Lighting Research and Technology, vol. 14, no. 3, pp. 151-159, 1982.

[3] P. Blaser and H. Dudli, "Tunnel lighting: method of calculating luminance of access zone L20," Lighting Research and Technology, vol. 25, no. 1, pp. 25-30, 1993.

[4] Commission Internationale de l'Éclairage (CIE), Guide for the Lighting of Road Tunnels and Underpasses, vol. 88 of CIE Publications, Vienna, Austria, 2004.

[5] Commission Internationale de l'Éclairage (CIE), Calculation of Tunnel Lighting Quality Criteria, vol. 189 of CIE Publications, Vienna, Austria, 2010.

[6] L. M. Gil-Martín, A. Peña-García, E. Hernández-Montes, and A. Espín-Estrella, "Tension structures: a way towards sustainable lighting in road tunnels," Tunnelling and Underground Space Technology, vol. 26, no. 1, pp. 223-227, 2011.

[7] A. Peña-García, L. M. Gil-Martín, A. Espín-Estrella, and F. Aznar-Dols, "Energy saving in road tunnels by means of transparent tension structures," in Proceedings of the International Conference on Renewable Energies and Power Quality (ICREPQ '10), Granada, Spain, 2010.

[8] E. Hernández-Montes, R. Jurado-Piña, and E. Bayo, "Topological mapping for tension structures," Journal of Structural Engineering, vol. 132, no. 6, pp. 970-977, 2006. 
[9] R. Jurado-Piña, L. M. Gil-Martín, and E. Hernández-Montes, "Topological mesh for shell structures," Applied Mathematical Modelling, vol. 33, no. 2, pp. 948-958, 2009.

[10] G. Parise, L. Martirano, and S. Pierdomenico, "An adaptive criterion to design the lighting system in the road tunnels," in Proceedings of the 42nd IEEE-IAS Annual Meeting, pp. 12441248, September 2007. 


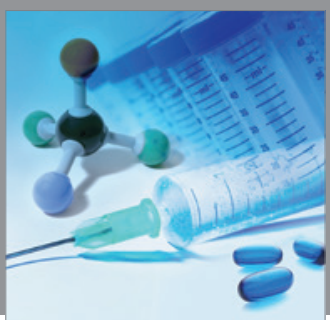

International Journal of

Medicinal Chemistry

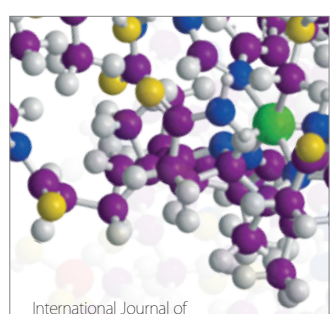

Carbohydrate Chemistry

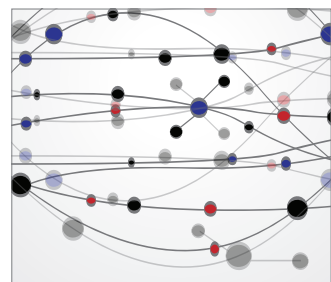

The Scientific World Journal
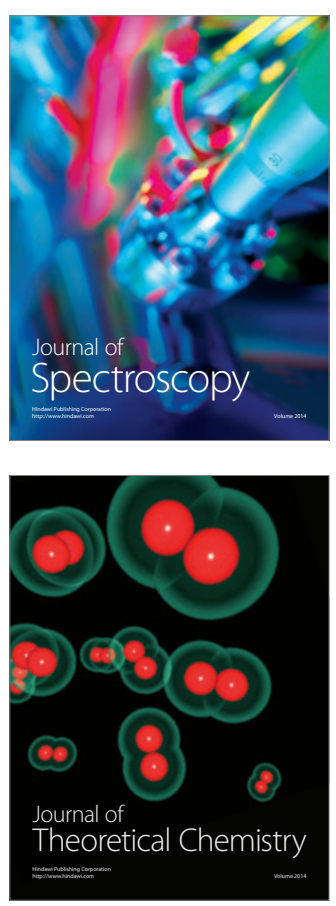
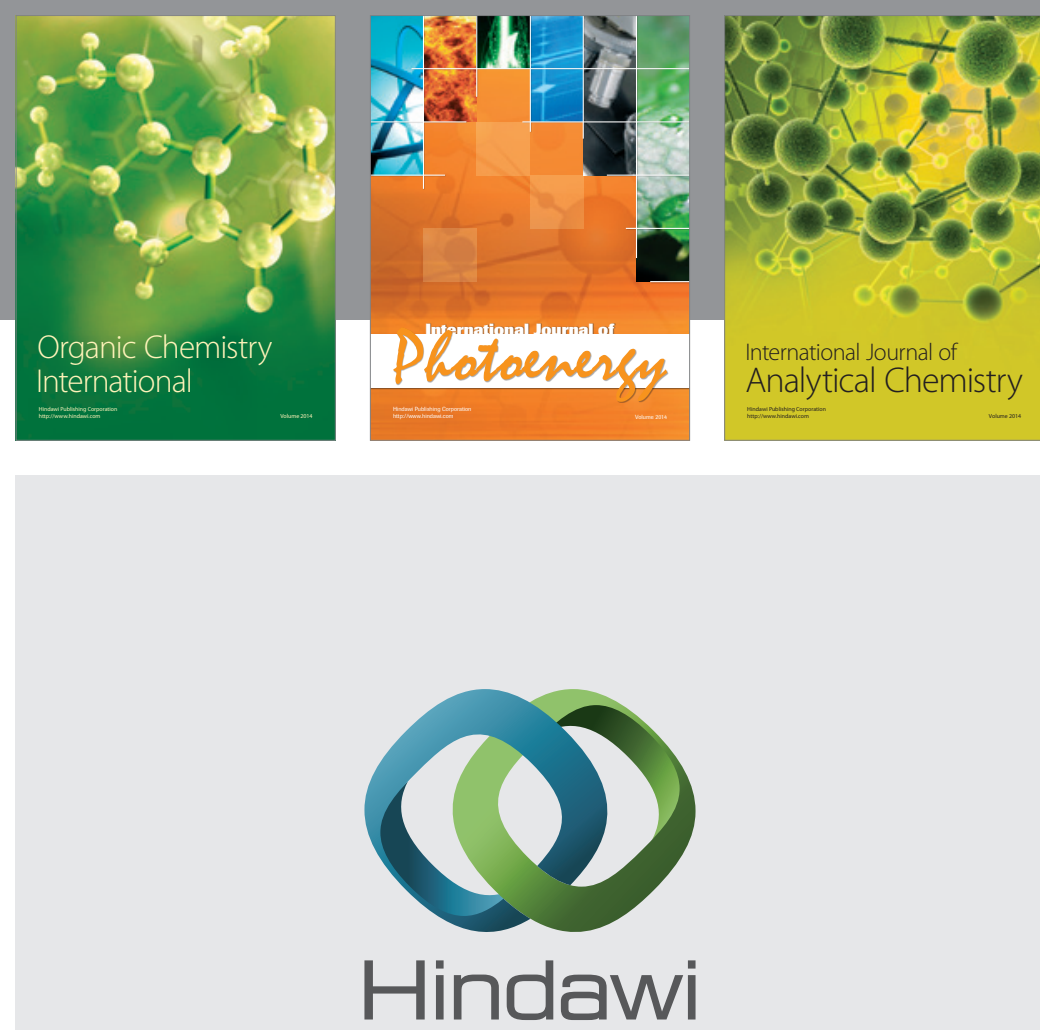

Submit your manuscripts at

http://www.hindawi.com
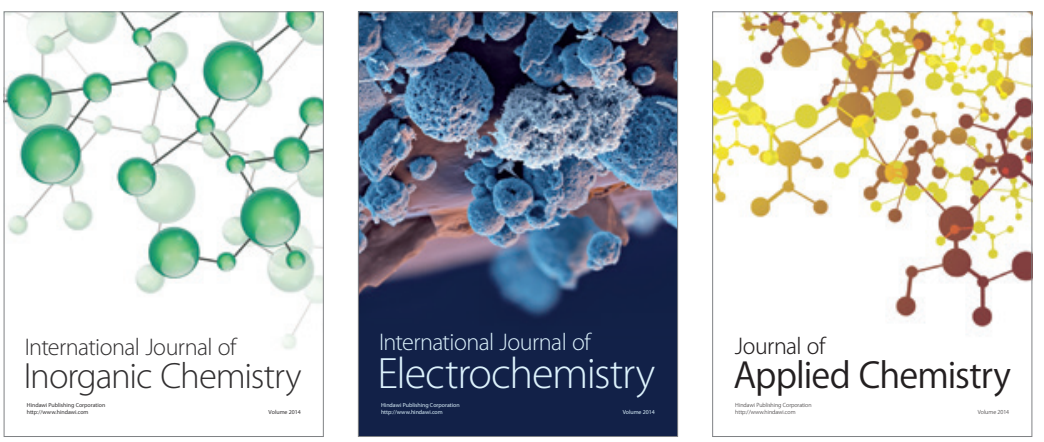

Journal of

Applied Chemistry
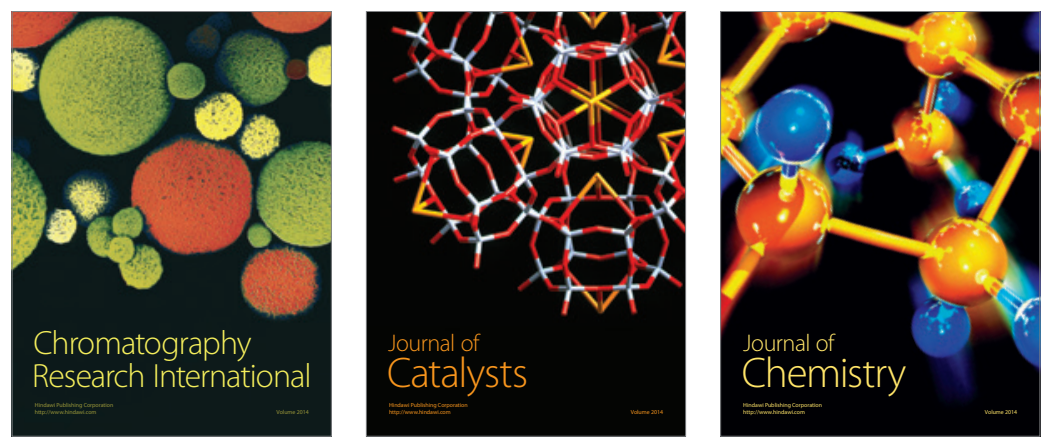
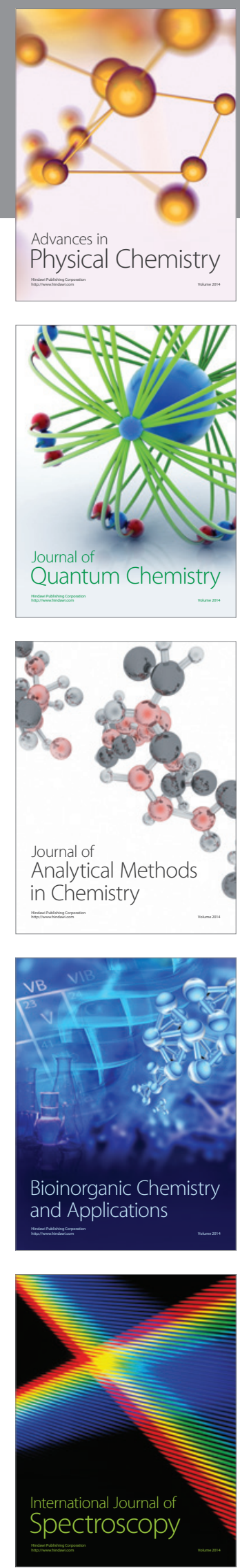